\title{
Influencia de los tratamientos de revenidos intercríticos en la concentración de níquel correspondiente a partículas de austenita residual en un acero con $9 \% \mathrm{Ni}^{(\bullet)}$
}

\author{
S. J. Casarin ${ }^{(*)}$, H-J. Kestenbach ${ }^{(*)}$ y E. Valencia ${ }^{(* *)}$
}

\begin{abstract}
Resumen En la estructura fina de aceros con $9 \% \mathrm{Ni}$, después de diferentes tratamientos térmicos de revenido a temperaturas entre $540-670{ }^{\circ} \mathrm{C}$ correspondiente al campo bifásico $(\alpha+\gamma)$ del diagrama $\mathrm{Fe}-\mathrm{Ni}$ se observaron partículas de dimensiones nanométricas de austenita residual. La estabilidad térmica de estas partículas resultó ser fuertemente influenciada por la concentración de níquel contenida en ellas. En este sentido fueron observados gradientes de concentración de níquel en las mismas dependiendo éstos del tiempo y la temperatura de revenido.
\end{abstract}

Palabras clave: Austenita residual. Acero 9 \% Ni. Revenido. Microanálisis.

\section{The influence of intercritical tempering treatment on the nickel concentration of retained austenite composition of a $9 \% \mathrm{Ni}$ steel}

\begin{abstract}
Retained austenite particles with nanometric dimensions in $9 \%$ nickel steels microstructures were observed after differents intercritical tempering treatments ranged between $540{ }^{\circ} \mathrm{C}$ and $670{ }^{\circ} \mathrm{C}$ corresponding to the $(\alpha+\gamma)$ two-phase field of Fe-Ni diagram. The thermal stability of particles turned out to be strongly influenced by its nickel contents and gradients of this element. The gradients were dependent on time and temperature of tempering.
\end{abstract}

Keyword: Retained austenite. $9 \% \mathrm{Ni}$ steel. Intercritical tempering treatment. Microanalysis.

\section{INTRODUCCIÓN}

La fase austenítica que se forma durante el tratamiento de revenido intercrítico después del temple en un acero al níquel, dentro de su campo bifásico $(\alpha+\gamma)$ según el diagrama $\mathrm{Fe}-\mathrm{Ni}$, aparece parcialmente retenida a la temperatura ambiente (1).

Se establece que las variaciones de la composición química afectan la estabilidad de la austenita. En el caso de los aceros con $9 \%$ Ni se confirma que la austenita inicial posee un alto contenido de carbono como resultado de la rápida difusión de

(•) Trabajo recibido el día 16 de junio de 1998 y aceptado en su forma final el día 31 de mayo de 1999.

(*) Dpto. de Eng. de Materias (DEMa) de la Universidad Federal de Sao Paulo (EFSCar), SP (Brasil).

(**) Universidad Central de las Villas (Cuba). este elemento y un contenido de níquel aproximadamente igual a la composición nominal $(9 \% \mathrm{Ni})$ de la aleación debido a la baja movilidad de los elementos sustitutivos. El prolongado mantenimiento de la temperatura de revenido logra una distribución de equilibrio de este elemento sustitutivo en la austenita formada (2).

Así, un factor que parece explicar la elevada estabilidad térmica de la austenita después del revenido, es la variación de la composición química en esta fase, enriquecida en carbono y níquel, conjuntamente con diferentes elementos de aleación secundarios presentes en el acero (2).

En la década de los 80 se comenzó a utilizar la técnica de microscopía electrónica de transmisión (MET) para determinar la composición química local de las pequeñas partículas de austenita. Kim y 
Morris Jr. (3) investigaron la austenita en aceros con $5,5 \% \mathrm{Ni}$, diseñados en la época como una alternativa más económica con relación a los aceros tradicionales con $9 \% \mathrm{Ni}$. Ningún análisis de carbono se podía efectuar por problemas de contaminación. Sin embargo, el contenido de níquel en la austenita residual después de los tratamientos térmicos resultó ser menor que el indicado por el diagrama de equilibrio en las aleaciones Fe-Ni. Los autores relacionaron estos bajos contenidos de níquel con la baja difusividad de este elemento $(0,01 \mu \mathrm{m}$ después de $1 \mathrm{~h}$ a $600^{\circ} \mathrm{C}$ ).

Kim y Morris Jr. (3) concluyeron que un análisis químico cuantitativo exacto de la austenita precipitada era poco fiable, mientras que un análisis cualitativo daba una tendencia de la variación de la composición química en función del tratamiento térmico. Sus resultados indicaron una acumulación de elementos tales como manganeso, cromo, níquel y molibdeno, mostrando un gradual enriquecimiento de níquel en las partículas después de un tratamiento prolongado de revenido a $600{ }^{\circ} \mathrm{C}$. Además, se puso de manifiesto que dos tratamientos de revenido consecutivos a $670{ }^{\circ} \mathrm{C}$ y $600{ }^{\circ} \mathrm{C}$, durante $1 \mathrm{~h}$, eran más efectivos para obtener la concentración adecuada de níquel y por tanto la estabilidad térmica de la partícula de austenita.

Fultz et al. (4) determinaron los contenidos locales de níquel y las concentraciones medias de carbono en la fase austenítica para aceros con $9 \% \mathrm{Ni}$, mostrándose que después de un revenido a $590{ }^{\circ} \mathrm{C}$ durante $81 \mathrm{~h}$ se lograban en las partículas de austenita residual contenidos cercanos al $15 \% \mathrm{Ni}$, próximos a los previstos por el diagrama binario Fe-Ni. En un trabajo anterior (5) estos autores habían observado que las partículas de austenita después de una hora de revenido a $600{ }^{\circ} \mathrm{C}$ se mantenían estables hasta una temperatura de $-196^{\circ} \mathrm{C}$.

Argumentos para explicar la pérdida de estabilidad térmica de la austenita residual con el aumento del tiempo y temperatura de revenido han sido expuestos por otros autores (6 y 7). En ellos se infiere una paulatina disminución de los contenidos de carbono y efectos debido a los cambios estructurales durante el tratamiento isotérmico (formación de martensita nueva y revenido de la misma).

Se informa (6) que un tratamiento simple de revenido en el campo $(\alpha+\gamma)$ a $600{ }^{\circ} \mathrm{C}$ sería suficiente para generar partículas estables de austenita hasta una temperatura criogénica en aceros comerciales con $9 \% \mathrm{Ni}$, temperatura de tratamiento también escogida por otros investigadores (5 y 8).

La investigación de la composición química de las partículas de austenita residual formadas durante el revenido merece así, la atención necesaria para precisar acerca de los mecanismos de su falta de estabilidad. El presente trabajo analiza la influencia de los diferentes regímenes de revenido intercrítico sobre la composición química (en particular la concentración de níquel) de las partículas de austenita residual a la temperatura ambiente.

\section{MATERIALES Y MÉTODOS}

Se utilizaron muestras de un acero comercial con 9 $\%$ Ni laminado en caliente, según una preparación industrial, y posteriormente enfriado al aire. Las probetas se templaron en agua desde una temperatura de $900{ }^{\circ} \mathrm{C}$ y su composición química fue la siguiente:

$$
\begin{gathered}
0,05 \% \mathrm{C}, 0,65 \% \mathrm{Mn}, 0,21 \% \mathrm{Si}, 9,6 \% \mathrm{Ni} \\
0,012 \% \mathrm{P}, 0,006 \% \mathrm{~S}, 0,038 \% \mathrm{Al}
\end{gathered}
$$

Con la finalidad de variar la cantidad, el tamaño y la composición química de la austenita residual en función de la temperatura y el tiempo de revenido, se calentaron muestras templadas del acero investigado, en estado totalmente martensítico, hasta una de las temperaturas correspondientes al campo bifásico $(\alpha+\gamma)$, a una velocidad de $20^{\circ} \mathrm{C} / \mathrm{min}$, en un horno eléctrico de pozo e inmersas en un baño de estaño. Las temperaturas y tiempos de permanencia en estos tratamientos fueron: $540{ }^{\circ} \mathrm{C}, 570^{\circ} \mathrm{C}$, $600{ }^{\circ} \mathrm{C}, 630^{\circ} \mathrm{C}$ y $670{ }^{\circ} \mathrm{C}$ durante $10 \mathrm{~min}, 1 \mathrm{~h}, 10 \mathrm{~h}$, y $100 \mathrm{~h}$. El control de la temperatura fue realizado por un termopar cromel-alumel tipo $K$ inmerso en el baño de estaño. La temperatura del horno osciló en $\pm 5^{\circ} \mathrm{C}$, mientras la del baño varió en $\pm 1{ }^{\circ} \mathrm{C}$ y el medio de enfriamiento fue el aceite.

Las muestras con el tratamiento de revenido fueron analizadas por difracción de rayos-X (DRX) para determinar las cantidades de fracciones de austenita residual a la temperatura ambiente tomando como referencia la norma ASTM E975-84.

Para la observación y microanálisis de las partículas de austenita residual en el microscopio electrónico de transmisión (MET-EDAX), se prepararon láminas finas en un Tenupol, utilizándose como electrólito una solución de $5 \%$ de $\mathrm{HClO}$ (ácido perclórico) y $95 \%$ de $\mathrm{CHCOOH}$ (ácido acético) a $7{ }^{\circ} \mathrm{C}$ de temperatura.

Las muestras se inclinaron a ángulos de $15^{\circ}$ para los ensayos de microanálisis al MET. El eje del detector para la radiación $\mathrm{X}$ tuvo un ángulo de inclinación constante de $20^{\circ}$ y el haz incidente sobre las partículas analizadas fue de un diámetro entre $50 \mathrm{a}$ $30 \mathrm{~nm}$. El tiempo de conteo para cada análisis fue de $100 \mathrm{~s}$.

En cada muestra se realizaron los análisis correspondientes a diferentes partículas lográndose un valor medio para las fracciones de concentración de níquel en las mismas. En aquellas partículas mayores se realizaron análisis en tres puntos diferentes, 
con el haz de diámetro $30 \mathrm{~nm}$, para verificar la existencia de algún gradiente de concentración del elemento analizado.

La unidad de detección del EDAX CM-120 consta de un detector de silicio en estado sólido, montado en un conjunto con alto vacío criogénico que posee una ventana patrón de berilio de espesor 7,5 $\mu \mathrm{m}$. Los resultados obtenidos por tal sistema (fracción de concentración de elementos químicos) dependen de algunos datos de entrada, entre los que se destacan el espesor del área analizada (muy variable) y la densidad del material. Los resultados pueden variar de acuerdo con el modelo escogido para el cálculo y, en el caso del EDAX CM-120, el modelo patrón fue el de Zaluzek (9). No obstante su aplicación requiere el conocimiento del espesor del área analizada.

Para superar los inconvenientes anteriores el mejor método está en convertir las intensidades integradas de los rayos $\mathrm{X}$ en porcentaje en peso de los elementos, utilizando una relación entre las intensidades integradas conocida como método de CliffLorimer (9)

$$
\frac{C_{i}}{C_{j}}=\mathrm{K}_{i j}\left(\frac{I_{i}}{I_{j}}\right)
$$

Donde: $C_{i}, C_{j}=$ fracción en peso de los elementos $i$ $\mathrm{y} j$

$I_{i}, I_{j}=$ intensidades integradas de las líneas de rayos $\mathrm{x}$ de los elementos $i$ y $j$

$\mathrm{K}_{i j}=$ constante.

Según algunos autores (9 y 10), dado $\mathrm{K}_{i j}$ para una selección particular del elemento de referencia $j$, es más conveniente adoptar el elemento solvente que para el acero estudiado fue el hierro y de tal forma $\mathrm{K}_{i j}=\mathrm{K}_{i \mathrm{Fe}}$.

La composición química de las partículas de austenita residual puede ser determinada a partir de la condición

$$
C_{i}+C_{j}=1-c^{\prime}
$$

donde $c^{\prime}$ es la suma de las concentraciones (\% en peso) de los elementos secundarios ( $\mathrm{Mn}, \mathrm{Si}, \mathrm{Cr}, \ldots$ ), fuera del intervalo de análisis, los cuales son conocidos o estimados.

En este caso:

$$
\begin{gathered}
\frac{C_{\mathrm{Ni}}}{C_{\mathrm{Fe}}}=\mathrm{K}_{\mathrm{NiFe}}\left(\frac{I_{\mathrm{Ni}}}{I_{\mathrm{Fe}}}\right) \\
C_{\mathrm{Ni}}+C_{\mathrm{Fe}}+c^{\prime}=1
\end{gathered}
$$

La precisión del método puede variar entre 1 y 4 $\%$, dependiendo de los errores en el cálculo de la constante $\mathrm{K}_{i j}$. Algunos valores de $\mathrm{K}_{i j}$, mas específi- camente los $\mathrm{K}_{\mathrm{AFe}}$ están tabulados para varios elementos químicos (10). El valor de $\mathrm{K}_{\mathrm{NiFe}}$ utilizado en el trabajo resultó ser $\mathrm{K}_{\mathrm{NiFe}}=1,102$ para una tensión de operación de $120 \mathrm{kV}$ según el modelo de Powell. Este valor calculado es apenas 0,5\% mayor que el valor tabulado $\left(\mathrm{K}_{\mathrm{NiFe} \text { (Tabulado) }}=1,096\right)$.

\section{RESULTADOS}

El material de entrega presentó una microestructura caracterizada por una martensita acicular con residuos de la estructura de laminación, que se mantuvieron después de todos los tratamientos térmicos de revenido realizados, lo que indica que la temperatura de recristalización es superior a las temperaturas utilizadas.

La necesidad de un revenido doble en el acero comercial con $9 \% \mathrm{Ni}$ para obtener una cantidad satisfactoria de austenita residual se hace dudosa y nula, pues con un revenido de apenas una hora, a temperaturas entre $570{ }^{\circ} \mathrm{C}$ y $630^{\circ} \mathrm{C}$, se ha demostrado que es posible obtener una fracción de austenita residual entre $8 \%$ y $10 \%$, suficiente para garantizar en el material una determinada estabilidad y tenacidad en condiciones criogénicas (1).

La figura 1, obtenida a partir de los ensayos de difracción de rayos- $X$, muestra el crecimiento de la cantidad de austenita residual a $600{ }^{\circ} \mathrm{C}$ con el aumento del tiempo de permanencia en la temperatura de revenido, apreciándose un incremento considerable del tiempo para aumentar el contenido entre 10-15\%.

La figura 2 a muestra una región de alta densidad de partículas de austenita residual en una muestra del acero con $9 \% \mathrm{Ni}$, revenida a $600{ }^{\circ} \mathrm{C}$ durante una hora, donde se aprecia la nucleación y crecimiento de las partículas según las fronteras de las ripas martensíticas.

Las partículas de austenita residual de mayores dimensiones, como las de la figura $2 \mathrm{~b}$, presentan un gradiente de concentración de níquel que influye de manera significativa en su estabilidad térmica. En estas partículas la concentración de níquel es menor en su interior aumentando hacia las regiones periféricas. Esto hace que la transformación $\gamma \rightarrow \alpha^{\prime}$ (martensita) se inicie en el interior de la partícula por ser la región más pobre en níquel y más enriquecida en carbono (4).

El enriquecimiento de solutos sustitutivos tales como níquel, manganeso y silicio en las partículas de austenita residual se ilustra en la figura 3. Esta figura muestra claramente la tendencia hacia una concentración de equilibrio en función del tiempo de permanencia a la temperatura de tratamiento térmico, hasta un valor de saturación en la partícula. Repetidas mediciones de la concentración de níquel en estas condiciones, realizadas a través del sistema EDAX-CM120 del microscopio electrónico (TEM), confirman esta tendencia, según indica la figura 4. 

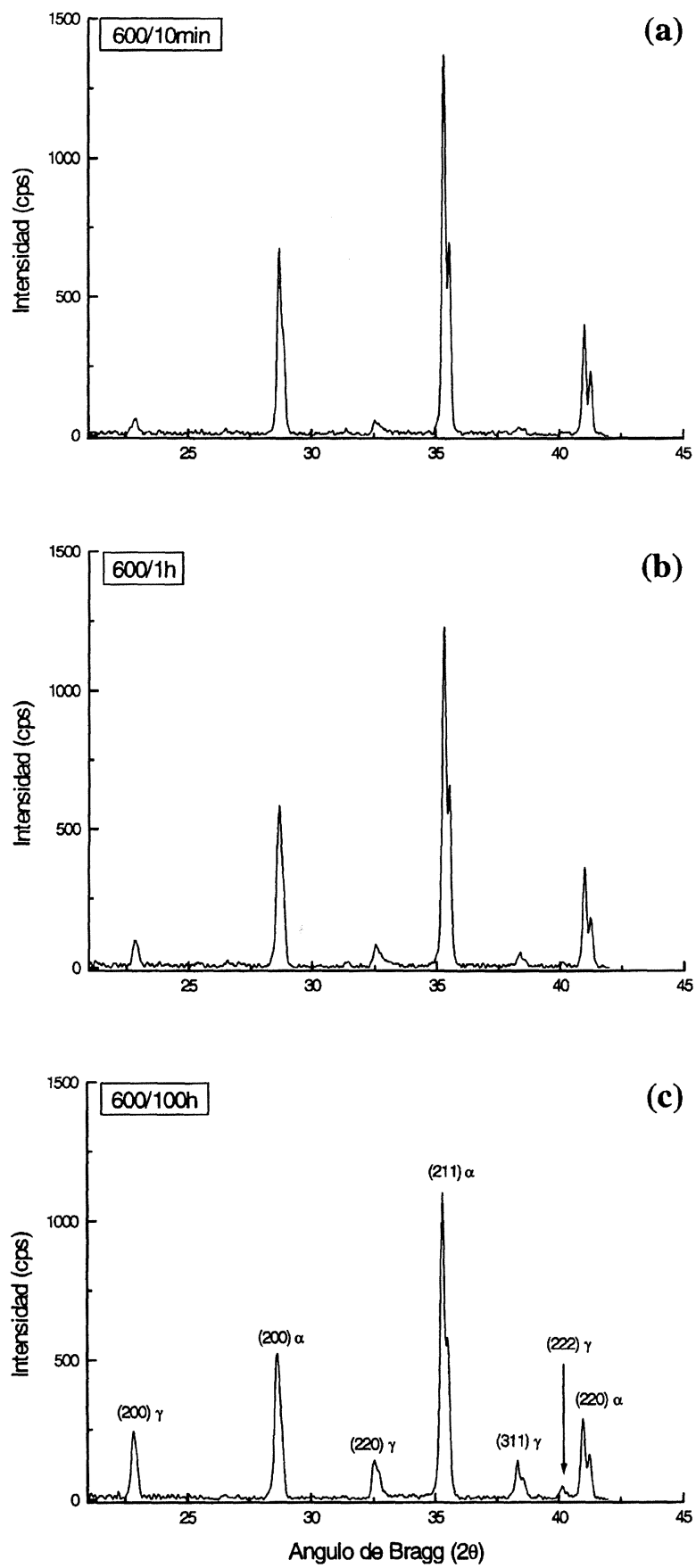

Fig. 1.- Difracción de rayos-X en el acero con $9 \% \mathrm{Ni}$, revenido a $600{ }^{\circ} \mathrm{C}$, muestra el crecimiento de la cantidad de austenita residual con el tiempo de permanencia a la temperatura de tratamiento térmico. (a) $\gamma_{\mathrm{r}}=5,4 \%$, (b) $\gamma_{\mathrm{r}}=8,1 \%$, (c) $\gamma_{\mathrm{r}}=15,7 \%$.

FIG. 1.-X-ray diffraction patterns of a tempered $9 \%$ Ni steel at $600^{\circ} \mathrm{C}$ showing the increasing retained austenite fraction with the increasing holding time temperature at the heat treatment temperature. (a) $\phi_{r}=5,4 \%$, (b) $\gamma_{r}=8,1 \%$, (c) $\gamma_{r}=15,7 \%$.

La figura 5 muestra la variación de la concentración de níquel en las partículas de austenita residual en función de la temperatura del tratamiento térmi- co, para un tiempo de permanencia a la temperatura de revenido de una hora. Los resultados de esta figura concuerdan con las observaciones de Kim y Morris Jr. (3), según las cuales, repetidas mediciones de un conjunto considerable de partículas dentro del rango de tratamientos térmicos intercríticos de temperatura, muestran un valor medio de la concentración de níquel cercano a lo establecido por el diagrama Fe-Ni (11-13).

\section{DISCUSIÓN}

\subsection{Tratamientos térmicos de revenido intercrítico}

Los resultados obtenidos concuerdan con las observaciones de algunos investigadores (4), según los cuales, revenidos por tiempos muy prolongados a $600{ }^{\circ} \mathrm{C}$ o revenidos a temperaturas mayores son perjudiciales para la estabilización de la austenita. De esta manera, en función de las velocidades de difusión del carbono y del níquel, revenidos realizados a temperaturas menores de $600{ }^{\circ} \mathrm{C}\left(540{ }^{\circ} \mathrm{C}\right)$ o mayores $\left(670{ }^{\circ} \mathrm{C}\right)$ afectan directamente la concentración de estos elementos (y de otros secundarios como el silicio y el manganeso) en las partículas de austenita, afectando su estabilidad. A la temperatura de $600{ }^{\circ} \mathrm{C}$ (Fig. 1) el tiempo de permanencia necesario para alcanzar una cantidad próxima al $15 \%$ de austenita residual es excesivamente elevado $(100 \mathrm{~h})$. Paralelamente, a las más altas temperaturas, la concentración de níquel en las partículas de austenita residual es insuficiente para garantizar su estabilidad ya que, a mayores temperaturas, menor es la solubilidad del níquel en esta fase.

Uno de los principales factores que pudiera afectar la estabilidad de la austenita residual en el acero con $9 \% \mathrm{Ni}$ lo constituye el tratamiento térmico de revenido. En condiciones comerciales este material sufre dos revenidos a dos temperaturas diferentes, (primero a $670{ }^{\circ} \mathrm{C}$ y después a $570{ }^{\circ} \mathrm{C}$ ) mantenido durante 17 minutos en ambos casos (14). Este procedimiento tiene como finalidad la obtención de una fracción volumétrica de austenita residual mayor al $10 \%$, contenido necesario para garantizar una excelente tenacidad en condiciones criogénicas (1).

En el presente trabajo el acero con $9 \% \mathrm{Ni}$ fue sometido a un único tratamiento de revenido. Los resultados de difracción de rayos X (Fig. 1) mostraron una fracción de austenita residual de hasta un $15,7 \%$ para un tratamiento a $600^{\circ} \mathrm{C}$ durante $100 \mathrm{~h}$. Comercialmente, una permanencia tan prolongada $(100 \mathrm{~h})$ de revenido no es económicamente realizable. Sin embargo, con un tratamiento más corto (1 h), la fracción de austenita residual alcanzada osciló entre $8 \%$ y $10 \%$, a temperaturas entre $570{ }^{\circ} \mathrm{C}$ y $630{ }^{\circ} \mathrm{C}$, considerada razonable para garantizar una 
adecuada estabilidad y tenacidad en condiciones criogénicas (1). Lo anterior constituye una alternativa económicamente viable en condiciones comerciales para los tratamientos de revenido en las aleaciones con $9 \% \mathrm{Ni}$.

La aparición de partículas de austenita residual es observable en casi todas las condiciones de tratamiento térmico. Tales partículas precipitan y crecen en las fronteras de granos y en los contornos de las ripas de martensita, quienes constituyen los principales caminos de difusión de los átomos sustitutivos como el níquel. Estas partículas son de tamaños variados, están orientadas en la dirección de laminación y tienen longitudes comprendidas entre 0.1 $\mu \mathrm{m}$ y $1 \mu \mathrm{m}$ y espesores que oscilaron entre $50 \mathrm{~nm}$ y $100 \mathrm{~nm}$. Como observaron otros autores (5), durante el afinamiento por pulimiento mecánico anterior al ataque electrolítico, muchas partículas de austenita residual pueden ser transformadas a martensita como consecuencia de la deformación plástica, disminuyendo por tanto la cantidad de las mismas observadas al MET.
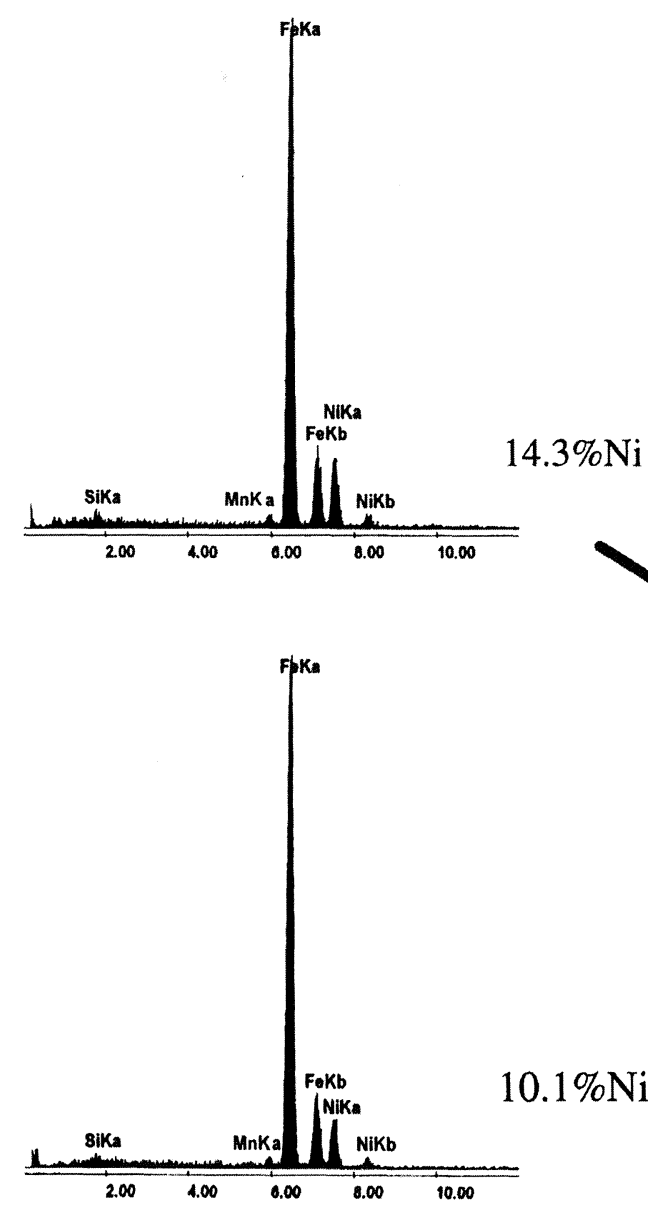

(b)

\subsection{Microanálisis de las partículas de austenita residual}

Los resultados del microanálisis de las partículas de austenita residual merecen consideraciones importantes. El primer aspecto se refiere a la gran dispersión de los resultados, correspondientes a los contenidos de níquel en las partículas, para las diferentes condiciones de tratamientos térmicos (Figs. 4 y 5).

La figura 4 muestra también que un aumento en el tiempo a la temperatura de revenido provoca un incremento en la concentración de níquel de las partículas de austenita residual, siendo la difusión

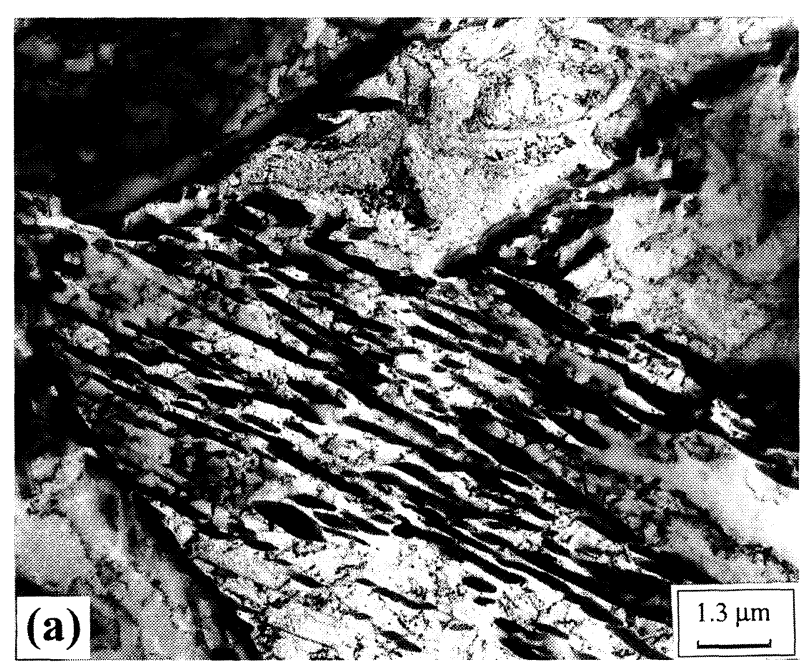

(a)

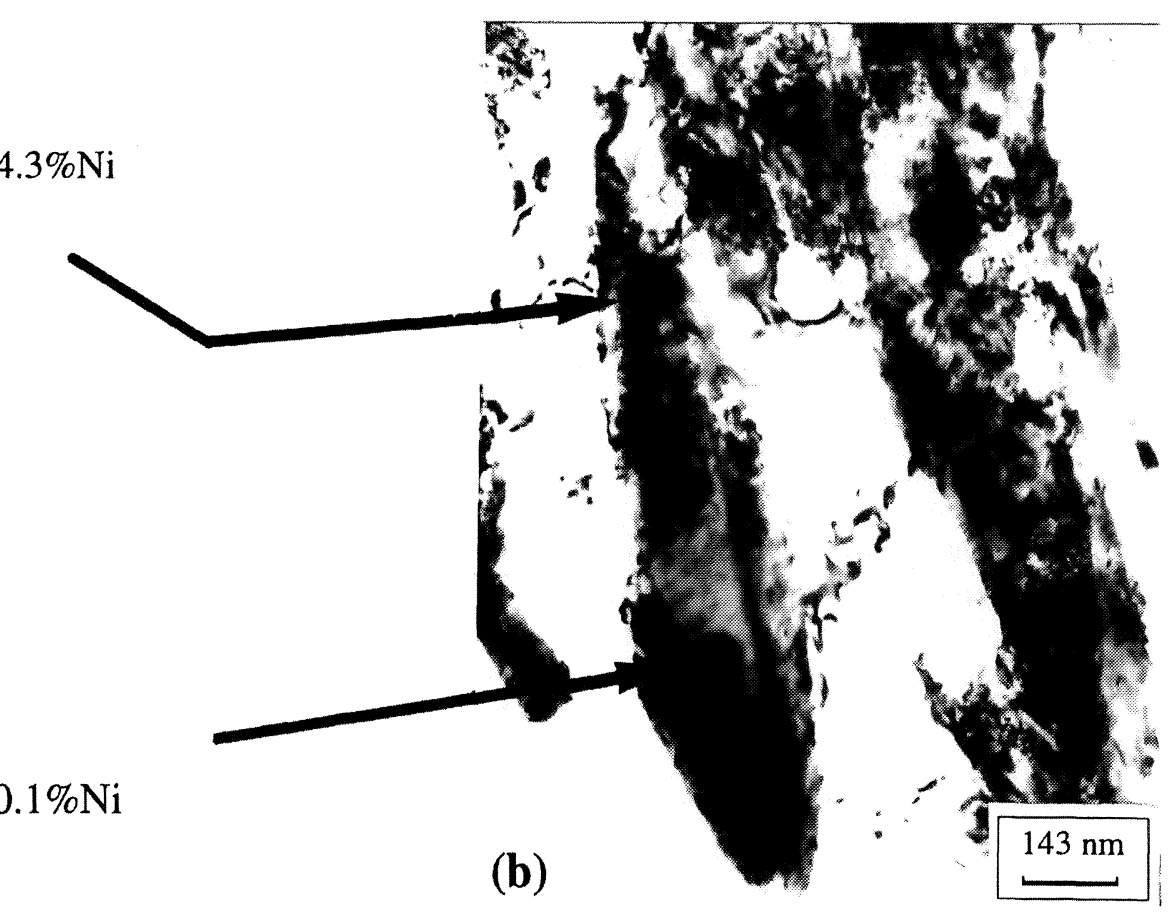

FIG. 2.- (a) Partículas de austenita residual distribuidas en la microestructura del acero con $9 \% \mathrm{Ni}$, revenido a $600{ }^{\circ} \mathrm{C}$ por $1 \mathrm{~h}$. (b) Partícula de austenita residual, ampliada, correspondiente a la misma muestra.

FIG. 2.- Retained austenite particles distributes in the $9 \%$ Ni steel microstructure tempered at $600{ }^{\circ} \mathrm{C}$ during $1 \mathrm{~h}$. (b) Retained austenite particle magnified from the same sample. 


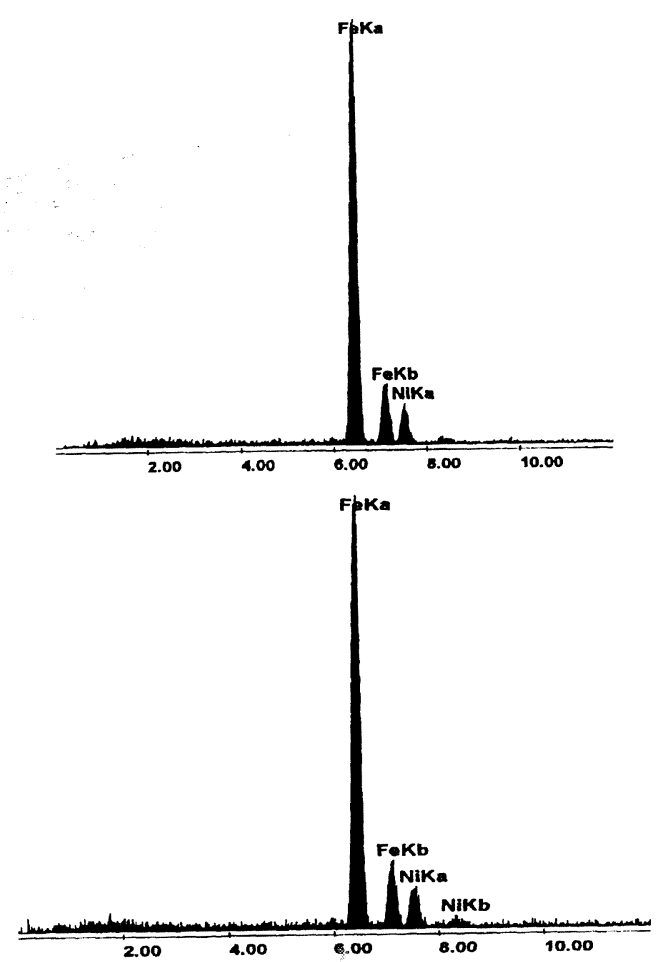

$10 \mathrm{~min}$

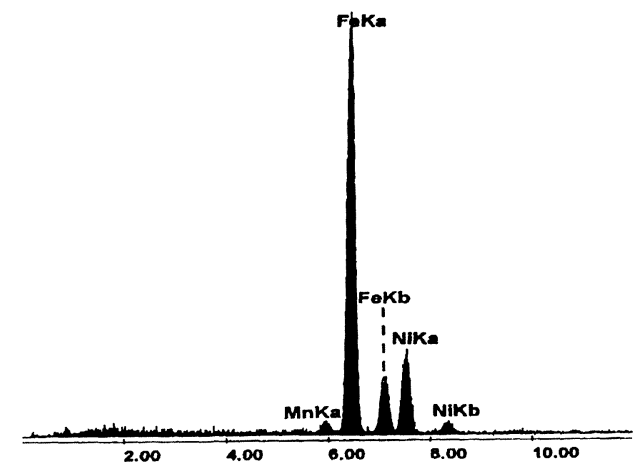

1 hora

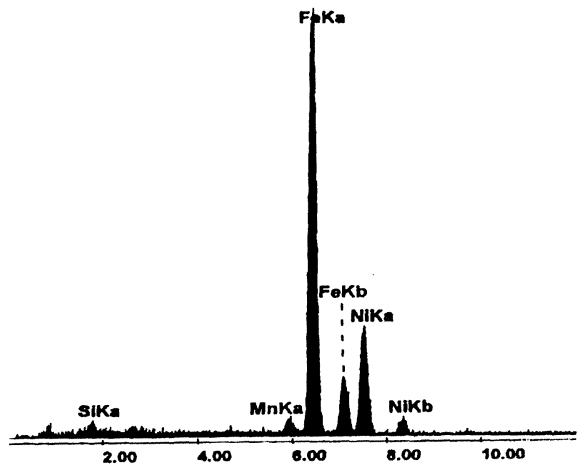

10 hs

$100 \mathrm{hs}$

FIG. 3.- Variación de la concentración de níquel en las partículas de austenita residual en función de la variación del tiempo de permanencia a la temperatura de revenido $\left(540{ }^{\circ} \mathrm{C}\right)$.

FIG. 3.- Changes in nickel content in retained austenite particles as a function of the holding time at the tempering temperature $\left(540^{\circ} \mathrm{C}\right)$.

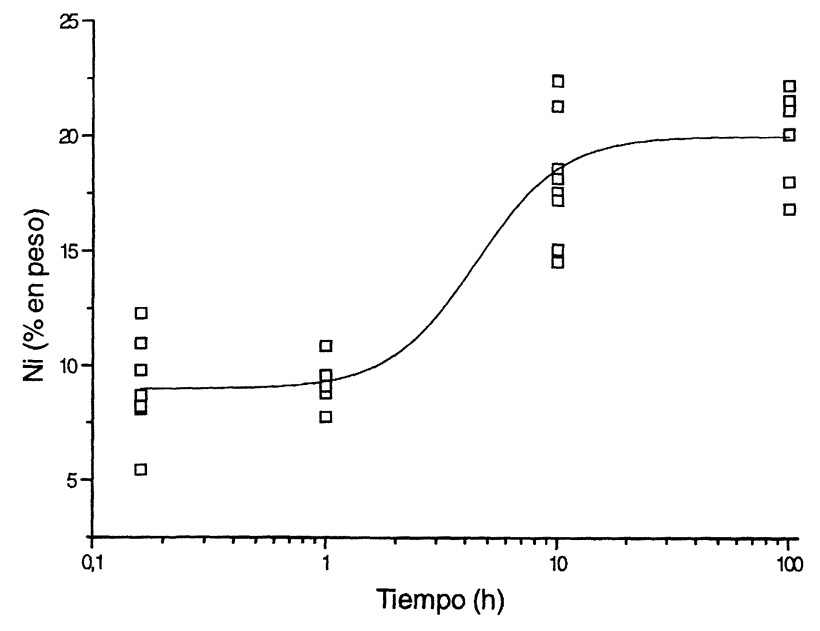

FIG. 4.- Variación de la concentración de níquel de las partículas de austenita residual para una temperatura de revenido de $540{ }^{\circ} \mathrm{C}$ a diferentes tiempos de tratamiento correspondientes a un acero con $9 \% \mathrm{Ni}$.

FIG. 4.- Changes in nickel content of the retained austenite particles of a $9 \% \mathrm{Ni}$ steel tempered at $540{ }^{\circ} \mathrm{C}$ during different holding times.

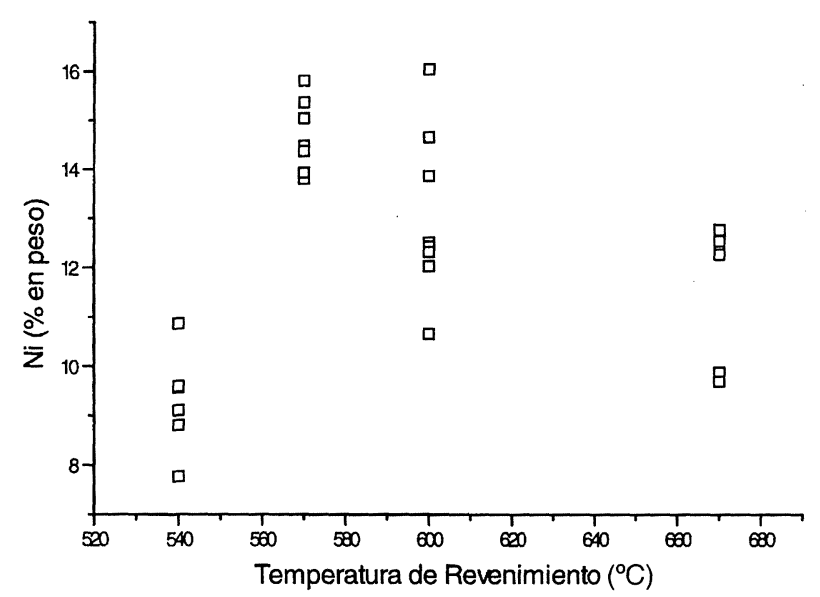

FIG. 5.- Variación de la concentración de níquel en las partículas de austenita residual en un acero con $9 \% \mathrm{Ni}$ en función de la temperatura de revenido para $1 \mathrm{~h}$ de permanencia.

FIG. 5.- Changes in nickel content of the retained austenite parfticles of a $9 \% \mathrm{Ni}$ steel as a function of tempering temperature kept $1 \mathrm{~h}$ at these temperatures.

el factor predominante, aunque también el vàlor de la temperatura de tratamiento afecta la concentración de níquel.

La variación en la composición de la austenita con el tratamiento térmico se puede interpretar, dada la baja difusividad de los elementos sustitutivos, en particular el níquel (3). Con el aumento de 
la temperatura de revenido (Fig. 5), la difusión del níquel se facilita, así como también la de otros elementos tales como el manganeso y el silicio; y como muestra el diagrama $\mathrm{Fe}-\mathrm{Ni}$, una elevación superior (mayor de $670^{\circ} \mathrm{C}$ ) de esta temperatura disminuirá la solubilidad de este elemento en la fase austenítica, decreciendo su estabilidad térmica.

Se han observado gradientes de concentración de níquel en las partículas de austenita residual (3, 4,12 y 13). El centro de las partículas formadas está enriquecido en carbono respecto al contenido original en la austenita (4), mientras que los contenidos de níquel son inferiores; por otro lado, en la periferia ocurre todo lo contrario, pues la concentración de níquel resultó ser superior. Se supone que la concentración de carbono disminuyó significativamente, lo que concuerda con el perfil de concentraciones de níquel medidas en las partículas de austenita residual (Fig. 2b). Como consecuencia de esta situación, durante el enfriamiento se transformará a martensita, primeramente la parte central y posteriormente las zonas periféricas.

Teniendo en cuenta las figuras 1 y 5 , el mejor rango de temperaturas para el tratamiento de revenido en un acero con $9 \% \mathrm{Ni}$ es entre 570 y $630^{\circ} \mathrm{C}$, con el objetivo de obtener las mayores fracciones de austenita residual, ya que en este intervalo es donde se obtiene una cantidad de austenita retenida y una concentración de níquel en las partículas suficiente para garantizar su estabilidad térmica.

No fue posible, en el presente trabajo, lograr una correlación entre la estabilidad térmica de la austenita residual en condiciones criogénicas y su composición química. Para un mejor estudio de la estabilidad térmica de la austenita residual es necesario considerar también la influencia de la concentración de carbono, de elementos de aleación secundarios (Mn y Si) y de la morfología de las partículas de austenita residual (3). Se observó que las partículas crecen preferencialmente en la dirección longitudinal de laminación y en menor cantidad según la dirección transversal (espesor), tal vez porque el níquel tiende a difundirse mejor a través de los contornos de granos, concentrándose en las extremidades de las partículas. Como la difusión intragranular del níquel es menor, el crecimiento en espesor es menos apreciable.

\section{CONCLUSIONES}

Mediante revenidos, cuya duración fue simplemente de una hora en el acero con $9 \% \mathrm{Ni}$ a temperatura entre $570{ }^{\circ} \mathrm{C}$ y $630^{\circ} \mathrm{C}$, se demostró que es posible obtener una fracción de austenita residual entre $8 \%$ y 10 $\%$, suficiente para garantizar la estabilidad térmica, dado el enriquecimiento de níquel en las mismas.

Se constató un gradiente de concentración en las partículas de austenita residual. La concentración de níquel aumenta desde el centro hasta la periferia en las partículas, así como también crece la concentración de elementos de aleación secundarios (Mn y $\mathrm{Si})$ con la prolongada permanencia a las temperaturas de revenido ensayadas. Esto se confirma porque las transformaciones $\gamma_{(\mathrm{fcc})} \rightarrow \alpha_{(\mathrm{bcc})}$ comienzan por el interior de las partículas.

Se destaca que con el aumento de la temperatura de revenido, la concentración de níquel en las partículas de austenita residual se aproxima a las mostradas en el diagrama de equilibrio Fe-Ni.

Una conclusión similar se obtiene para una temperatura fija de tratamiento térmico $\mathrm{y}$, variando el tiempo de permanencia en ésta, la concentración de níquel en las partículas de austenita tiene un valor de equilibrio, también concordante con el diagrama binario $\mathrm{Fe}-\mathrm{Ni}$.

\section{Agradecimientos}

Los autores están agradecidos al apoyo financiero de FAPESP (Fundación de Ayuda a la Investigación del Estado de São Paulo), por los procesos núms. 96/09523-9 y 94/0101-9. A CNPq por el proceso 301080/96-8.

\section{REFERENCIAS}

(1) Marschall, C.W., Hehemann, R.F. y Troiano, A.R. Trans. ASM, 55, 1962: 135-148.

(2) KIM, K.J. y SchwarTZ, J.H. Mater. Sci. Eng., 33, 1978: 5-20.

(3) KIM, J.I. y MoRris JR., J.W. Metal. Trans. A, 12A, (11), 1981: 1957-1963.

(4) Fultz, B., Kim, J.I., KIM, Y. Hy Morris JR. J.W. Metal. Trans. A, 17A, (6), 1986: 967-972.

(5) Fultz, B., Kim, J.I., KIM, Y.H., KIM, H.J., FrioR, G.A. y Morris JR. J.W. Trans. A, 16A, (12), 1985: 2237-2249.

(6) KIM, J.I., SYN, C.K. y Morris JR. J.W. Metal. Trans. A, 14A, (1), 1983: 93-103.

(7) Fultz, B. y Morris JR., J.W. Metal. Trans. A, 16A, (12), 1985: 2251-2256.

(8) Kinaka, R., Furukimi, O., Kubo, T., Okumura, T., Shingyoji, M. y Kudo, J. Kawasaki Steel Technical Report, 17, 1987: 64-72.

(9) Newbury, D.E., Joy, D.C., Echlin, P., Fiori, C.E. y GoldsteIn, J.I. Advanced Scanning Electron Microscopy and X-Ray Analysis. Plenum Press, second edition, 1987.

(10) Joy, D.C., Romig JR., A.D. y Goldstein, J.I. Principles of Analytical Electron Microscopy. Plenum Press, second edition, 1989.

(11) Metals Handbook. Metallography, Structure and Phase Diagrams. 8th edition, 8, ASTM, Metals Park, Ohio, 1973.

(12) Romig JR., A.D. y Goldstein, J.I. Metal. Trans. A, 11A, (7), 1980: 1151-1159.

(13) Romig JR., A.D. y Goldstein, J.I. Metal. Trans. A, 9A, (11), 1978: 1599-1609.

(14) Faria, A.V., Barros, H.N.O., Américo, F.L.A. y RatnaPULI, R.C. Metal. \& Mater. ABM, 49, (414), 1993: 121-126. 